\title{
A CONSTRUCTION RELATED TO THE COSINE PROBLEM
}

\author{
MIHAIL N. KOLOUNTZAKIS
}

(Communicated by J. Marshall Ash)

\begin{abstract}
We give a constructive proof of the fact that for any sequence of positive integers $n_{1}, n_{2}, \ldots, n_{N}$ there is a subsequence $m_{1}, \ldots, m_{r}$ for which

$$
-\min _{x} \sum_{1}^{r} \cos m_{j} x \geq C N
$$

where $C$ is a positive constant. Uchiyama previously proved the above inequality with the right-hand side replaced by $C \sqrt{N}$. We give a polynomial time algorithm for the selection of the subsequence $m_{j}$.
\end{abstract}

Notation. Let $C$ denote an arbitrary positive constant and $\mathbf{N}=\{1,2,3, \ldots\}$ be the set of positive integers. For any real $t$ we write $t^{-}=\min \{0, t\}$. We denote by $\lfloor t\rfloor$ the greatest integer not greater than $t$ and by $\lceil t\rceil$ the smallest integer not smaller than $t$.

The Cosine Problem. Chowla [2] conjectured that for any distinct positive integers $n_{1}, \ldots, n_{N}$

$$
-\min _{x} \sum_{j=1}^{N} \cos n_{j} x \geq C \sqrt{N}
$$

(There are sequences $n_{j}$ for which the above minimum is at most $C \sqrt{N}$ in absolute value.) The best result known today in this direction is that of Bourgain [1], who proved that

for some $\varepsilon>0$.

$$
-\min _{x} \sum_{j=1}^{N} \cos n_{j} x \geq C 2^{\log ^{e} N}
$$

Uchiyama [4] proved that there is always a subsequence $m_{1}, \ldots, m_{r}$ of $n_{1}, \ldots, n_{N}$ for which

$$
-\min _{x} \sum_{j=1}^{r} \cos m_{j} x \geq C \sqrt{N}
$$

He actually proved the stronger statement

$$
\frac{1}{2 \pi} \int_{0}^{2 \pi}\left|\sum_{j=1}^{r} \cos m_{j} x\right| d x \geq C \sqrt{N} .
$$

Received by the editors March 15, 1993.

1991 Mathematics Subject Classification. Primary 42A05; Secondary 68Q99. 
In this paper we improve (1).

Theorem 1. For any sequence $n_{1}, \ldots, n_{N}$ of positive integers there is a subsequence $m_{1}, \ldots, m_{r}$ such that

$$
-\min _{x} \sum_{j=1}^{r} \cos m_{j} x \geq C N .
$$

Theorem 1 is an obvious corollary of the following more general theorem.

Theorem 2. Let $w_{k} \geq 0$ and $w=\sum_{1}^{\infty} w_{k}<\infty$. Then there is a set $E$ of positive integers for which

$$
-\min _{x} \sum_{k \in E} w_{k} \cos k x \geq C w .
$$

The essential content of this paper is that the proof of Theorem 2 (and consequently of Theorem 1) we give is constructive. Indeed, there is a simple nonconstructive proof of our theorem.

Proof of Theorem 2-Nonconstructive (Odlyzko [3]). Define

$$
f(x)=\sum_{1}^{\infty} w_{k}(\cos k x)^{-} \text {. }
$$

Then

$$
\begin{aligned}
\frac{1}{2 \pi} \int_{0}^{2 \pi} f(x) d x & =\sum_{k=1}^{\infty} w_{k} \frac{1}{2 \pi} \int_{0}^{2 \pi}(\cos k x)^{-} d x \\
& =-\frac{1}{\pi} w .
\end{aligned}
$$

Thus there is $x_{0} \in[0,2 \pi)$ with $f\left(x_{0}\right) \leq-\frac{1}{\pi} w$. Let $E=\left\{k \in \mathbf{N}: \cos k x_{0} \leq 0\right\}$. Then obviously

$$
\sum_{k \in E} \cos k x_{0} \leq-\frac{1}{\pi} w .
$$

We now give a constructive proof of Theorem 2 with a worse constant. (See Remark 1 after the proof for the exact meaning of the word "constructive".)

We shall need two lemmas.

Lemma 1. Let $I_{k}=\left(a_{k}, b_{k}\right) \subseteq(0,1), k=1,2, \ldots$, be intervals of length at least $\theta>0$ and $w_{k}$ be nonnegative weights associated with them. Let also $w=\sum_{1}^{\infty} w_{k}<\infty$. Then there is an interval $J \subseteq(0,1)$, with $|J|=\theta / 2$, for which

$$
\sum_{J \subseteq I_{k}} w_{k} \geq \frac{1}{2} \theta w .
$$

Proof of Lemma 1. Let $m=\lfloor 2 / \theta\rfloor$ and $J_{\nu}=[\nu \theta / 2,(\nu+1) \theta / 2)$, for $\nu=$ $0,1, \ldots, m-1$. Write also $s_{\nu}=\sum_{a_{k} \in J_{\nu}} w_{k}$. Since $w=\sum_{0}^{m-2} s_{\nu}$, there is some $\nu_{0} \leq m-2$ for which

$$
s_{\nu_{0}} \geq \frac{w}{m-1} \geq \frac{1}{2} \theta w .
$$

Let $J=J_{\nu_{0}+1}$. Then $J$ satisfies (7) since $a_{k} \in J_{\nu_{0}}$ implies $J_{\nu_{0}+1} \subseteq I_{k}$.

The following lemma is a useful special case of Theorem 2. 
Lemma 2. Let $a>0, \sigma>1, \rho \geq 24 \sigma$,

$$
E_{j}^{\prime}=\left[\rho^{j} a, \sigma \rho^{j} a\right) \cap \mathbf{N}
$$

for $j=0,1,2, \ldots$, and $E^{\prime}=\bigcup_{j=0}^{\infty} E_{j}^{\prime}$. Assume also $w_{k} \geq 0, w=\sum_{1}^{\infty} w_{k}<$ $\infty$, and $w_{k}=0$ outside $E^{\prime}$. Then there is a set $E \subseteq E^{\prime}$ for which

$$
-\min _{x} \sum_{k \in E} w_{k} \cos k x \geq \frac{1}{48 \sigma} w .
$$

Proof of Lemma 2. First observe that in any interval of length at least $2 \pi / k$ there is a subinterval of length $2 \pi / 12 k$ in which $\cos k x \leq-1 / 2$. According to this observation, for all $k \in E_{0}^{\prime}$ there is an interval $I_{k}$ contained in $(0,2 \pi / a)$, of length at least $2 \pi / 12 \sigma a$, in which $\cos k x \leq-1 / 2$. By Lemma $1(\theta=$ $1 / 12 \sigma)$ there is an interval $J_{0} \subseteq(0,2 \pi / a)$ of length $2 \pi / 24 \sigma a$ for which

$$
\sum_{J_{0} \subseteq I_{k}} w_{k} \geq \frac{1}{24 \sigma} \sum_{k \in E_{0}^{\prime}} w_{k}
$$

Let $E_{0}=\left\{k \in E_{0}^{\prime}: J_{0} \subseteq I_{k}\right\}$. Then

$$
\sum_{k \in E_{0}} w_{k} \cos k x \leq-\frac{1}{48 \sigma} \sum_{k \in E_{0}^{\prime}} w_{k}, \quad \text { for all } x \in J_{0} .
$$

Similarly we can find an interval $J_{1} \subseteq J_{0}$, with $\left|J_{1}\right|=2 \pi / 12 \sigma \rho a$, and $E_{1} \subseteq E_{1}^{\prime}$, such that

$$
\sum_{k \in E_{1}} w_{k} \cos k x \leq-\frac{1}{48 \sigma} \sum_{k \in E_{1}^{\prime}} w_{k}, \quad \text { for all } x \in J_{1} .
$$

This is possible since $\rho \geq 24 \sigma$ and therefore $J_{0}$ is big enough to accomodate all frequencies in $E_{1}^{\prime}$. In the same fashion we define $J_{2} \supseteq J_{3} \supseteq \cdots$ and $E_{2}, E_{3}, \ldots$. Finally we set $E=\bigcup_{0}^{\infty} E_{j}$. It follows that (8) is true.

We can now complete the proof of the theorem.

Proof of Theorem 2-Constructive. Let $\sigma=2, \rho=64$, and write for $\nu=$ $0, \ldots, 5$

$$
A_{\nu}=\sum_{j=0}^{\infty}\left[\rho^{j} \sigma^{\nu}, \rho^{j} \sigma^{\nu+1}\right) \cap \mathbf{N} .
$$

Since $\mathbf{N}=\bigcup_{0}^{5} A_{\nu}$, there is some $\nu_{0}$ for which

$$
\sum_{k \in A_{\nu_{0}}} w_{k} \geq \frac{1}{6} w
$$

An application of Lemma 2 with $\sigma=2, \rho=64, a=1$, and the collection of $w_{k}$ for $k \in A_{\nu_{0}}$ furnishes a set $E \subseteq A_{\nu_{0}}$ for which

$$
-\min _{x} \sum_{k \in E} w_{k} \cos k x \geq \frac{1}{6 \cdot 48 \cdot 2} w
$$

Remarks. 1. The simple proof of Theorem 1 mentioned can of course be made constructive by looking for an $x$ that satisfies

$$
\sum_{1}^{N}\left(\cos n_{k} x\right)^{-} \leq-\frac{1}{2 \pi} N
$$


among the points $x_{j}=j h$, for $j=0, \ldots,\lfloor 1 / h\rfloor$. But $h$ has to be smaller than $C n_{N}^{-1}$, and this leads to an algorithm which in the worst case takes time exponential in the size of the input (which is considered to be the number of binary digits required to write down all $\left.n_{1}, \ldots, n_{N}\right)$. For example, if $n_{N}=2^{N}$, then the algorithm needs time at least $C 2^{N}$, but the size of the input is at most $N^{2}$.

In contrast, our construction takes time which is polynomial in the size of the input (in other words, polynomial in $N \log n_{N}$ ). Assume that we are given $N$ positive integers $n_{1} \leq \cdots \leq n_{N}$, and let $L=\left\lfloor\log _{2} n_{N}\right\rfloor$. Define $w_{j}=\mid\{k \in$ $\left.\mathbf{N}: j=n_{k}\right\} \mid$. The algorithm we described consists of the following steps. The notation of Lemma 2 is used throughout.

(1) Find for which $\nu_{0} \in\{0, \ldots, 5\}$ inequality (11) is true.

(2) Construct the sequence of intervals $J_{0} \supseteq J_{1} \supseteq \ldots$ and the sequence of sets $E_{0}, E_{1}, \ldots$. This proceeds inductively. Having constructed the interval $J_{m-1}$ and the set $E_{m-1}$ we

(a) construct the intervals $I_{n_{k}}$ for all $n_{k} \in E_{m}^{\prime}$,

(b) find (as described in Lemma 1) a subinterval $J_{m}$ of $J_{m-1}$ which is big and is contained in many of the $I_{n_{k}}$ 's. The set $E_{m}$ consists of those $n_{k} \in E_{m}^{\prime}$ for which $J_{m} \subseteq I_{n_{k}}$.

Notice that the sequences $J_{m}$ and $E_{m}$ have length $O(L)$.

After observing that we never need to perform arithmetic with more than $O(L)$ binary digits, it is easy to see that all the above can be carried out in time $O\left(N \cdot L^{2}\right)$, since an algebraic operation on two numbers, with $O(L)$ binary digits each, takes $O\left(L^{2}\right)$ time.

2. Uchiyama's proof of equation (1) is probabilistic. We give an even simpler constructive proof of equation (1). (Of course, Uchiyama proved the stronger statement in equation (2) about the $L^{1}$ norm of a subseries.) Assume $n_{1} \leq$ $n_{2} \leq \cdots \leq n_{N}$, and let $\rho$ be any fixed number between 2 and 3 , say $\rho=5 / 2$. Observe that if $n_{N} \leq \rho n_{1}$, then

$$
-\min _{x} \sum_{j=1}^{N} \cos n_{j} x \geq C N,
$$

as can be seen by evaluating the function $\sum_{j=1}^{N} \cos n_{j} x$ for $x=(\pi / 2+\varepsilon) / n_{1}$, where $\varepsilon=\varepsilon(\rho)$ is a small positive constant.

Let $\lambda_{1}=n_{1}$, and define $\lambda_{k} \in\left\{n_{1}, \ldots, n_{N}\right\}$ recursively by

$$
\lambda_{k}=\min \left\{n_{j}: n_{j}>\rho \lambda_{k-1}\right\} \cup\left\{n_{N}\right\} .
$$

Let $L$ be the length of the sequence $\lambda_{k}$; that is, let $\lambda_{L}$ be the first $\lambda$ equal to $n_{N}$. Then either $L \geq \sqrt{N}$ or there is some $k$ for which the set

$$
A=\left\{n_{j}: \lambda_{k} \leq n_{j}<\lambda_{k+1}\right\}
$$

has more than $\sqrt{N}$ elements. In the first case we have

$$
-\min _{x} \sum_{j=1}^{L-1} \cos \lambda_{j} x \geq C L \geq C \sqrt{N},
$$


since the $\lambda_{j}$ 's form a lacunary sequence with ratio $\rho>2$. Otherwise, according to the above observation, we have

$$
-\min _{x} \sum_{n_{j} \in A} \cos n_{j} x \geq C|A| \geq C \sqrt{N},
$$

which completes the proof.

3. It is easy to see that Theorem 2 holds also for complex $w_{k}$, with $w=$ $\sum\left|w_{k}\right|<\infty$ and writing $e^{i k x}$ in place of $\cos k x$. Also the minimum in (4) has to be interpreted as the minimum (or maximum) of the real part.

\section{REFERENCES}

1. J. Bourgain, Sur le minimum d'une somme de cosinus, Acta Arith. 45 (1986), 381-389.

2. S. Chowla, Some applications of a method of A. Selberg, J. Reine Angew. Math. 217 (1965), 128-132.

3. A. M. Odlyzko, personal communication.

4. S. Uchiyama, On the mean modulus of trigonometric polynomials whose coefficients have random signs, Proc. Amer. Math. Soc. 16 (1965), 1185-1190.

Department of Mathematics, Stanford University, Stanford, California 94305

Current address: Institute for Advanced Study, Olden Lane, Princeton, New Jersey 08540 